\title{
riccafd
}

Revista Iberoamericana de Ciencias de la Actividad Física y el Deporte

\section{REALIDAD DE LAS ECUACIONES PREDICTIVAS PARA PRESCRIBIR EJERCICIO SEGÚN FRECUENCIA CARDÍACA MÁXIMA EN PACIENTES CON OBESIDAD}

\author{
REALITY OF PREDICTIVE EQUATIONS TO PRESCRIBE \\ EXERCISE ACCORDING TO MAXIMUM CARDIAC \\ FREQUENCY IN PATIENTS WITH OBESITY
}

Pereira-Rodríguez, Javier Eliecer ${ }^{1}$; Peñaranda-Florez, Devi Geesel²;

Pereira-Rodríguez, Pedro3; Pereira-Rodríguez, Ricardo4; Flores-Rodríguez, Julio5; Marin-Herrera, Luis ${ }^{6}$.

Grupo de investigación Aletheia (Instituto Profesional de Terapias y Humanidades).

${ }^{1}$ Fisioterapeuta, Especialista en Rehabilitación Cardiopulmonar, Maestrante en Ciencias de la Salud, Maestrante en Innovación Educativa. Instituto Profesional de Terapias y Humanidades. Puebla, México.jepr87@hotmail.com

${ }^{2}$ Fisioterapeuta, Especialista en Neurorehabilitación, Magister en dificultades del aprendizaje. Independiente. Puebla, México. devipeflo@gmail.com

${ }^{3-4}$ Médico general. Independiente. Cúcuta, Colombia.

pedro k1333@hotmail.com; ricardo16pereira@hotmail.com

${ }^{5-6}$ Fisioterapeutas en formación. Instituto Profesional de Terapias y Humanidades. Puebla, México. floresjulio431@gmail.com; louisherrera1998@gmail.com

Correspondencia: Pereira-Rodríguez, Javier Eliecer.jepr87@hotmail.com

Código UNESCO: 241106. Fisiología del Ejercicio

Consejo de Europa: 14. Fisioterapia y rehabilitación

Recibido el 4 de noviembre de 2018

Aceptado el 16 de abril de 2019

DOI: http://dx.doi.org/10.24310/riccafd.2019.v8i2.6453

\section{RESUMEN}

Introducción y objetivos: La prescripción del ejercicio tiene como finalidad mejorar los resultados en el ejercicio, y se debe prescribir según las características y Frecuencia cardiaca máxima (FCM) del paciente.

Métodos y materiales: Estudio observacional, descriptivo y transversal con 67 participantes, de edad promedio 35 $\pm 12,6$ años. Se obtuvó antropometría, signos vitales, escala de Borg, cuestionario para factores de riesgo cardiovascular (FRC) y realización de prueba de esfuerzo.

Resultados: En prueba de esfuerzo la FCM de 172,82+18,81 lpm. Las ecuaciones 
con menor diferencia, para la población total y el genero masculino, fue la formula Morris, para el genero femenino fue la ecuación 210-edad. Para los participantes con menos de 4 FRC fue Astrang y para los participantes con mas de 4 FRC fue 210-Edad.

Conclusiones: Para pacientes con obesidad no se recomienda la FCM a través de las ecuaciones evaluadas, debido que varían según múltiples variables, especialmente 220-Edad y Tanaka.

Palabras clave: frecuencia cardiaca, cardiología, prueba de esfuerzo, ejercicio.

\section{ABSTRACT}

Introduction and objectives: The prescription of the exercise aims to improve the results in the exercise, and should be prescribed according to the characteristics and maximum heart rate (FCM) of the patient.

Methods and materials: Observational, descriptive and cross-sectional study with 67 participants, average age $35 \pm 12.6$ years. Anthropometry, vital signs, Borg scale, questionnaire for cardiovascular risk factors (CRF) and exercise test were obtained.

Results: In an effort test the FCM of $172.82 \pm 18.81 \mathrm{bpm}$. The equations with the least difference, for the total population and the male gender, was the formula Morris, for the female gender was the 210-age equation. For participants with less than 4 FRC was Astrang and for participants with more than 4 FRC it was 210-Age.

Conclusions: For patients with obesity, FCM is not recommended through the equations evaluated, because they vary according to multiple variables, especially 220 Age and Tanaka.

Key words: heart rate, cardiology, stress test, exercise.

\section{INTRODUCCIÓN}

El exceso de peso corporal de origen graso (obesidad), hoy en día es uno de los problemas más significativos de la salud pública a nivel mundial, esto debido a la gran magnitud de personas que la padecen, provocando en ellos ciertos factores no saludables ${ }^{1}$.

La Obesidad, es una enfermedad crónica, sistémica y multicausal, la cual puede presentarse a cualquier edad, en distintas etnias y en todas las clases sociales. Además, constituye el sexto factor principal de riesgo de defunción en el mundo, ya que cada año fallecen globalmente alrededor de 3.4 millones de personas adultas $^{1-2}$.

Ahora bien, el aumento excesivo del peso corporal es un proceso paulatino que normalmente inicia en la infancia y la adolescencia, esto a consecuencia de un desequilibrio entre la ingesta y el gasto energético; que posterior a esto, se ve reflejado en un aumento de peso. Como resultado de estudios epidemiológicos, se propone que las causas principales de esta enfermedad se relacionan con ciertos factores genéticos, ambientales y a diversos estilos de vida. Desde 1998, la Organización Mundial 
de la Salud considera a la obesidad como una epidemia global, que establece un importante problema de salud pública ${ }^{1,3-4}$, con un aproximado de 1,700 millones de adultos con sobrepeso y 312 millones presentan obesidad 5 .

Respecto a las comorbilidades, la obesidad no influye de la misma manera en el desarrollo de las diversas comorbilidades, ya que se predisponen más en unas que en otras. Entre las que se asocian a la obesidad, se destacan aquellas dependientes de alteraciones metabólicas asociados a esta condición, como la Diabetes Mellitus (DM2), dislipidemia aterogénica, hígado graso y síndrome ovárico poliquístico. Otras dependen de los cambios físico-mecánicos del exceso de peso, como la hipoventilación, apnea nocturna y osteoartrosis ${ }^{6-7}$. Todo lo anterior, más otras afectaciones multisistemicas generan una sobrecarga a nivel cardiovascular generadora a largo plazo de una falla cardiaca (Grafico 1), que debe ser identificada a tiempo y de ser necesario abarcar un tratamiento oportuno. Es por ello, que las pruebas de esfuerzo juegan un papel predominante en el diagnostico y prescripción del ejercicio del paciente con obesidad.

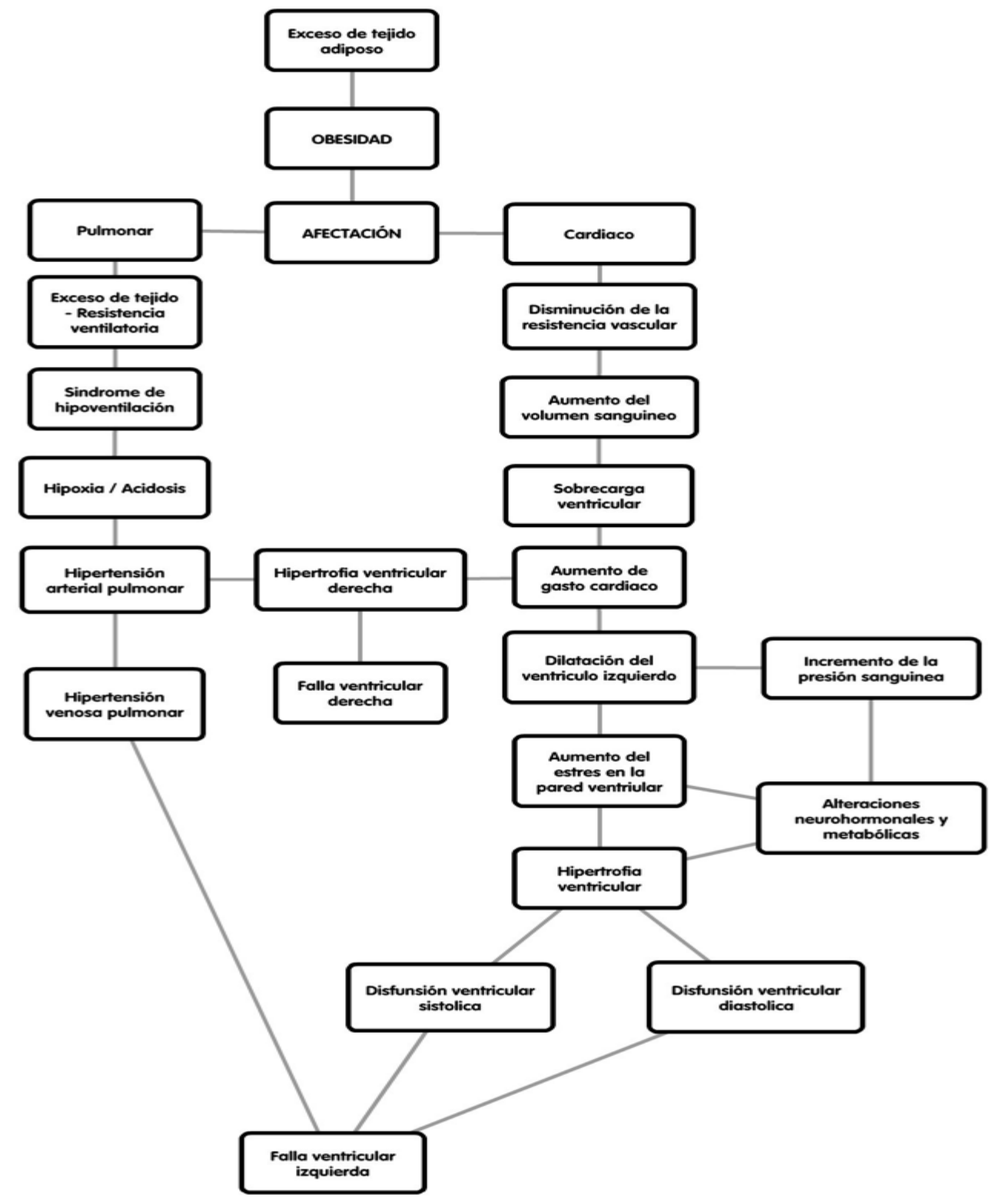

Gráfico 1. Asociación de la obesidad con la falla cardiaca. 
La Prueba de esfuerzo (PE), es un procedimiento diagnóstico en la cual se evalúa la respuesta cardiovascular a un ejercicio físico progresivo. Es una de las exploraciones cardíacas más utilizadas, ya que evidencia con fines diagnósticos o pronósticos alteraciones que no se hacen presentes en reposo. Así como, valorar la capacidad física que presenta la persona ${ }^{8-9}$.

Según el tipo de PE, como lo es en este caso: la banda sin fin o tapiz rodante, se emplean algunos protocolos del cual destaca el de Bruce, con 7 etapas de 3 minutos de duración cada una, en la cual cada etapa va aumentando tanto la velocidad, como la inclinación del tapiz rodante; este protocolo suele ser bien tolerado y aplicado a personas de edad media y con las capacidades necesarias para realizar una actividad física normal, aunque por otra parte, las inclinaciones superiores al $20 \%$ han sido difíciles de alcanzar ${ }^{10-11}$.

Ahora bien, en el caso de personas mayores o con una capacidad física muy inferior, el protocolo que más se utiliza en ese aspecto; es el de Naughton, que a diferencia del protocolo de Bruce, este se realiza por etapas de 2 minutos en el que progresivamente va aumentando la inclinación del tapiz rodante, pero no la velocidad ${ }^{11}$. No obstante, independientemente de su metodología ambos protocolos son eficaces para determinar la frecuencia cardíaca máxima (FCM) del paciente. Siendo esta, una de las formas de control fisiológico más frecuente en la evaluación de la intensidad del esfuerzo, a la que el organismo se somete durante un ejercicio.

Según Bouzas-Marins et al. (2010)12 proponen como estrategia una lista de ecuaciones predictivas para determinar la FCmáx, tomando como parámetro indispensable el índice de masa corporal (IMC) como un factor determinante en la respuesta de la FCmáx; respecto a esto, surgen diferentes autores con otras variables, que afirman una mayor eficacia para determinar la FCmáx de los pacientes. Por lo tanto, luego de revisar la literatura disponible e investigaciones recientes sobre dicho tema. Surge como pregunta de investigación, ¿Qué tan válidas y efectivas son las ecuaciones para determinar la frecuencia cardíaca máxima en los pacientes con obesidad? Y por ende, se plantea como objetivo principal: Comparar la FCmáx de una prueba de esfuerzo frente a los resultados de las ecuaciones predictivas en pacientes con obesidad.

\section{MATERIALES Y METODOS}

Se realizó un estudio observacional, descriptivo y transversal que cuantificó los factores de riesgo cardiovascular, antropometría, respuesta cardiovascular pre, peri y post prueba de esfuerzo; como también, comparación de dichos resultados frente a las ecuaciones predictivas para determinar la frecuencia cardiaca máxima en participantes con obesidad. Dicha investigación se realizó en una población de 67 (45 Mujeres y 22 Hombres) participantes con una edad promedio de 35,58 \pm 12,6 años de la ciudad de Cúcuta, Colombia. Donde los individuos fueron sometidos a un cuestionario, pruebas y medidas.

Para los criterios de inclusión se tuvo en cuenta que los pacientes debían ser mayor de 18 años de edad, tener un índice de masa corporal mayor a 30, pesar 
más de $65 \mathrm{Kg}$ y firmar un consentimiento informado avalado por el comité de ética de la institución.

Así mismo, en los criterios de exclusión los participantes no podían tener dolor en miembros inferiores, disnea y/o fatiga en reposo mayor a 3, no estar bajo medicación de betabloqueadores, individuos con alteraciones cardiovasculares o antecedentes quirúrgicos de este tipo y/o antecedentes de infarto agudo de miocardio. Como criterios de retiro, se tuvieron en cuenta la inestabilidad hemodinámica durante la prueba y la manifestación de no querer continuar.

Para la recolección de datos sociodemográficos se empleó un instrumento de creación propia, el cual fue diligenciado mediante un interrogatorio al paciente antes de la prueba de esfuerzo. Para hallar las variables morfológicas y antropométricas; se inicio determinando la talla del participante mediante el tallímetro Adult Acrylic Halter Wall Kramer 2104; para el peso, se utilizó la Balanza Tezzio Digital Balance TB-30037 (bioimpedancia eléctrica) y para la circunferencia abdominal (CA) la cinta métrica Asámico de $150 \mathrm{~cm} \mathrm{60"} \mathrm{Gree} \mathrm{con} \mathrm{una} \mathrm{precisión} \mathrm{de}$ $1 \mathrm{~mm}$ y tomando los referentes anatómicos descritos por Frisancho y los puntos de cohorte como parámetro diagnostico las cifras en hombres de $91 \mathrm{~cm}$ y mujeres de $89 \mathrm{~cm}$ en la CA, ambos expuestos por Buendía R. et al. ${ }^{13}$.

Respecto a la prueba de esfuerzo, fue realizada en una banda sin fin con el protocolo de Bruce. Los pacientes no podían ingerir bebidas, fumar ni consumir ningún tipo de droga o medicamento que pudiera interferir con la FCmáx. La disnea y esfuerzo percibido se valoraron mediante la escala de Borg modificada; la cual consiste en 10 indicadores del esfuerzo que percibe el individuo. Su uso en el área de las ciencias de la salud está dado para evaluación, ajustes de intensidad y/o cargas de trabajo.

La frecuencia cardíaca se obtuvo mediante el sistema Polar RS800CX Multisport, mientras que la saturación arterial de oxígeno con un oxímetro de pulso portátil (Nellcor Puritan Bennett); dichas mediciones fueron tomadas pre, peri y post prueba de esfuerzo. Referente a la toma de tensión arterial, esta fue obtenida de manera manual al inicio, final y luego de 5 minutos de haber finalizado la prueba de esfuerzo. El valor de la FCmáx obtenida después de un esfuerzo máximo se consideró como una variable dependiente (resultado) y como variables independientes (predictores) se tomaron las ecuaciones para determinar la FCmáx. Además, se resalta que el diseño y desarrollo de la investigación fue realizada bajo las consideraciones éticas de la Declaración de Helsinki y la Resolución No. 008430 de el Ministerio de Salud de Colombia.

\section{ANÁLISIS ESTADÍSTICO}

Para la descripción de las variables cuantitativas, fue necesario expresarlas como media aritmética y desviación estándar. Respecto a la comparación de la FCmáx entre las ecuaciones teóricas frente a la obtenida en la prueba de esfuerzo, se realizó mediante ANOVA (Análisis de la varianza de una vía) para comparar las diferencias de género y edad en las distintas variables estudiadas, además de pruebas post hoc, mediante la prueba de Tukey, para ver las diferen- 
cias entre los distintos grupos de edad y antropometría. En todos los casos se estableció el nivel de significación en el $5 \%(p<0,05)$ y todos los análisis fueron realizados en el programa Stata.

\section{RESULTADOS}

A continuación, se presentan los resultados de las variables estudiadas (Caracteristicas sociodemográficas, antropometría, factores de riesgo cardiovascular y respuesta hemodinámica) y su comparación con las ecuaciones predictivas para determinar la frecuencia cardíaca máxima.

En la Tabla 1, se muestran las características y factores de riesgo cardiovasculares de la población estudiada; la cual, estuvo conformada por 45 Mujeres y 22 Hombres ( $n=67)$. Resaltando que el $100 \%$ de la muestra fueron pacientes con obesidad, cuyos principales factores de riesgo cardiovasculares fueron el sedentarismo $(100 \%)$, dieta inadecuada $(61,11 \%)$, antecedentes familiares $(76,11 \%)$, alcoholismo (58.20\%) e hipertensión arterial $(28,36 \%)$ entre otros.

Tabla 1. Características de población.

\begin{tabular}{|c|c|c|c|c|c|c|}
\hline & Total & $\%$ & Masculino & $\%$ & Femenino & $\%$ \\
\hline $\begin{array}{l}\text { Genero } \\
\text { Edad }\end{array}$ & 67 & 100 & 22 & 32,84 & 45 & 32,84 \\
\hline Menores de 30 & 24 & 35,82 & 11 & 50 & 13 & 28,88 \\
\hline $\begin{array}{l}\text { Mayores de } 30 \\
\text { Etnia }\end{array}$ & 43 & 64,17 & 11 & 50 & 32 & 71,11 \\
\hline Blanco & 26 & 38,8 & 11 & 50 & 15 & 33,33 \\
\hline Mestizo & 38 & 56,71 & 9 & 40,9 & 29 & 64,44 \\
\hline $\begin{array}{l}\text { Afrocolombiano } \\
\text { Nivel educativo }\end{array}$ & 3 & 4,47 & 2 & 9,1 & 1 & 2,22 \\
\hline Primaria & 1 & 1,49 & 1 & 4,54 & 0 & 0 \\
\hline Secundaria & 9 & 13,43 & 4 & 18,18 & 5 & 11,11 \\
\hline Bachiller & 26 & 38,81 & 7 & 31,82 & 19 & 42,22 \\
\hline Téc. O Tecnol. & 4 & 5,97 & 1 & 4,54 & 3 & 6,67 \\
\hline Pregrado & 25 & 37,31 & 9 & 40,91 & 16 & 35,55 \\
\hline $\begin{array}{l}\text { Postgrado } \\
\text { Tabaquismo }\end{array}$ & 2 & 2,98 & 0 & 0 & 2 & 4,44 \\
\hline Exfumadores & 20 & 29.85 & 10 & 22.22 & 10 & 45.45 \\
\hline 3-7 Cigarrillos & 2 & 2.98 & 0 & 0 & 2 & 9.09 \\
\hline 9-15 Cigarrillos & 1 & 1.49 & 1 & 2.22 & 0 & 0 \\
\hline $\begin{array}{l}\text { No Consumen } \\
\text { Alcoholismo }\end{array}$ & 44 & 65.67 & 11 & 24.44 & 33 & 73.33 \\
\hline Consumidores & 39 & 58.20 & 13 & $59 \%$ & 26 & 57,77 \\
\hline $1 \times$ Semana & 16 & 23.88 & 7 & 31.81 & 9 & 20 \\
\hline 1 o 2 x Semana & 20 & 29.85 & 3 & 13.63 & 17 & 37.77 \\
\hline $2 \times$ Semana & 3 & 4.47 & 2 & 9.09 & 1 & 2.22 \\
\hline $4 \times$ Semana & 1 & 1.49 & 1 & 4.54 & 0 & 0 \\
\hline $\begin{array}{l}\text { No Consumen } \\
\quad \text { Dieta inadecuada }\end{array}$ & 27 & 40.29 & 9 & 40.90 & 18 & 40 \\
\hline $1 \mathrm{vez} \times$ Mes & 20 & 29.85 & 2 & 9.09 & 18 & 40 \\
\hline
\end{tabular}




\begin{tabular}{|c|c|c|c|c|c|c|}
\hline 1 vez $x$ Semana & 15 & 22.38 & 7 & 31.81 & 8 & 17.77 \\
\hline 2 vez x Semana & 12 & 17.91 & 3 & 13.63 & 9 & 20 \\
\hline 3 vez $x$ Semana & 11 & 16.41 & 2 & 9.09 & 0 & 0 \\
\hline 4 o mas $x$ Semana & 3 & 4.4 & 1 & 4.5 & 2 & 9.09 \\
\hline $\begin{array}{l}\text { No consumen } \\
\text { Hipertensión arterial }\end{array}$ & 15 & 22.38 & 7 & 31.81 & 8 & 17.77 \\
\hline $\mathrm{Si}$ & 19 & 28.36 & 6 & 27.27 & 13 & 28.88 \\
\hline $\begin{array}{l}\text { No } \\
\quad \text { Diabetes }\end{array}$ & 48 & 71.64 & 16 & 72.72 & 32 & 71.11 \\
\hline $\mathrm{Si}$ & 9 & 13.43 & 3 & 13.63 & 6 & 13.33 \\
\hline No & 58 & 86.56 & 19 & 86.36 & 39 & 86.66 \\
\hline Obesidad & & & & & & \\
\hline I & 37 & 55.22 & 16 & 72.12 & 21 & 46.67 \\
\hline "I Sedentarismo & 21 & 31.34 & 3 & 13.64 & 18 & 40 \\
\hline Si & 67 & 100 & 45 & 67.16 & 22 & 32.84 \\
\hline No & 0 & 0 & 0 & 0 & 0 & 0 \\
\hline
\end{tabular}

Por otra parte, en la tabla 2 se describe la respuesta hemodinámica de los participantes antes y después de una prueba de esfuerzo; encontrándose claramente una mayor FCmáx en las mujeres en comparación con los hombres $(173,9 \pm 17,5$ vs $168,9 \pm 22,1)$; además, se logró evidenciar las diferencias encontradas entre la FCmáx luego de una prueba de esfuerzo comparada con la obtenida mediante las ecuaciones predictivas (Tabla 3); y a su vez, diferenciar la frecuencia cardíaca máxima de los participantes con menos de 4 factores de riesgo cardiovascular versus con mas de $4(<4: 179,4 \pm 17,7$ vs $>4: 167,1 \pm 18,6)$ (Tabla 4$)$.

Tabla 2. Respuesta hemodinámica luego de una prueba de esfuerzo.

\begin{tabular}{lcccccccc}
\hline & \multicolumn{2}{c}{ Promedio } & \multicolumn{2}{c}{$\mathrm{DE}$} & \multicolumn{3}{c}{ Mínimo } & \multicolumn{2}{c}{ Máximo } \\
\hline & $\mathrm{H}$ & $\mathrm{M}$ & $\mathrm{H}$ & $\mathrm{M}$ & $\mathrm{H}$ & $\mathrm{M}$ & $\mathrm{H}$ & $\mathrm{M}$ \\
FC (pre) & 90,77 & 98,88 & 12,95 & 21,35 & 66 & 70 & 110 & 140 \\
FC (post) & 168,95 & 173,91 & 22,16 & 17,57 & 117 & 133 & 199 & 199 \\
FC 1 min. & 148,28 & 150,75 & 17,53 & 20,05 & 115 & 111 & 178 & 182 \\
FC 3 min. & 127,23 & 131,31 & 16,16 & 15,22 & 95 & 105 & 160 & 165 \\
FC 5 min. & 116,19 & 121,44 & 13,62 & 13,70 & 86 & 95 & 140 & 158 \\
SatO2 (pre) & 97,31 & 97,26 & 1,12 & 1,05 & 94 & 95 & 99 & 98 \\
SatO2 (post) & 91,31 & 95,57 & 1,57 & 1,77 & 92 & 90 & 98 & 98 \\
PAS (pre) & 125,18 & 120,44 & 13,80 & 13,89 & 100 & 97 & 160 & 160 \\
PAS (post) & 131,95 & 128,17 & 14,25 & 17,31 & 100 & 100 & 160 & 180 \\
PAD (pre) & 80,13 & 78,24 & 12,06 & 11,28 & 60 & 60 & 100 & 100 \\
PAD (post) & 83,50 & 79,35 & 6,49 & 11,26 & 75 & 60 & 100 & 100 \\
Disnea (pre) & 0,13 & 0,26 & 0,35 & 0,49 & 0 & 0 & 1 & 2 \\
Disnea (post) & 8,31 & 7,42 & 1,17 & 2,60 & 6 & 0 & 10 & 10 \\
\hline
\end{tabular}




$\begin{array}{llllllllc}\text { Fatiga (pre) } & 0,18 & 0,24 & 0,39 & 0,60 & 0 & 0 & 1 & 2 \\ \text { Fatiga (post) } & 8,36 & 7,66 & 1,43 & 2,27 & 6 & 1 & 10 & 10\end{array}$

FC: Frecuencia cardíaca; SatO2: Saturación arterial de oxigeno; PAS: Presión arterial sistólica; PAD: Presión arterial diastólica; FRC: Factores de riesgo cardiovascular; DE: Desviación estándar; H: Hombres; M: Mujeres.

Tabla 3. Comparación de FCM por prueba de esfuerzo frente a ecuaciones predictivas.

\begin{tabular}{|c|c|c|c|c|c|c|c|c|c|c|c|c|}
\hline & \multicolumn{2}{|c|}{ Población general } & \multicolumn{2}{|c|}{ Hombres } & \multicolumn{3}{|c|}{ Mujeres } & \multicolumn{5}{|c|}{ FORMULA VS PRUEBA DE ESFUERZO } \\
\hline & Promedio & DE & Promedio & DE & Promedio & DE & $\begin{array}{c}\text { Dif } \\
\text { General }\end{array}$ & Valor $p$ & $\begin{array}{l}\text { Dif. } \\
\text { Hombres }\end{array}$ & Valor $p$ & $\begin{array}{c}\text { Dif. } \\
\text { Mujeres }\end{array}$ & Valor $p$ \\
\hline Prueba de esfuerzo & 172,28 & 19,17 & 168,95 & 22,16 & 173,91 & 17,57 & & & & & & \\
\hline 220-Edad & 184,42 & 12,62 & 185,45 & 15,06 & 183,91 & 11,40 & 12,13 & 0,000 & 16,50 & 0,006 & 10,00 & 0,001 \\
\hline 210-Edad & 174,42 & 12,62 & 175,45 & 15,06 & 173,91 & 11,40 & 2,13 & 0,447 & 6,50 & 0,261 & 0,00 & 1 \\
\hline Tanaka (1997) $211-0,8^{*}$ Edad & 182,53 & 10,10 & 183,36 & 12,05 & 182,12 & 9,12 & 10,25 & 0,000 & 14,41 & 0,012 & 8,21 & 0,009 \\
\hline Tanaka (2001) 207-0,7"Edad & 182,09 & 8,84 & 182,82 & 10,54 & 181,74 & 7,98 & 9,81 & 0,000 & 13,86 & 0,013 & 7,83 & 0,011 \\
\hline Tanaka (2001) 206-0,7"Edad & 181,09 & 8,84 & 181,82 & 10,54 & 180,74 & 7,98 & 8,81 & 0,001 & 12,86 & 0,021 & 6,83 & 0,029 \\
\hline Tanaka (2001) 208,75-0.73"Edad & 182,78 & 9,21 & 183,53 & 10,99 & 182,41 & 8,32 & 10,49 & 0,000 & 14,58 & 0,010 & 8,50 & 0,007 \\
\hline Robinson (1938) $212-0,77^{*} \mathrm{Edad}$ & 184,60 & 9,72 & 185,40 & 11,59 & 184,21 & 8,78 & 12,32 & 0,000 & 16,45 & 0,004 & 10,30 & 0,001 \\
\hline Inbar $205,8-0,685^{\circ}$ Edad & 181,43 & 8,65 & 182,14 & 10,31 & 181,08 & 7,81 & 9,14 & 0,000 & 13,18 & 0,018 & 7,17 & 0,022 \\
\hline Astrang $211-0,922^{*}$ Edad & 178,19 & 11,64 & 179,15 & 13,88 & 177,73 & 10,51 & 5,91 & 0,054 & 10,19 & 0,091 & 3,82 & 0,293 \\
\hline Bruce $210-0,662^{*}$ Edad & 186,44 & 8,36 & 187,13 & 9,97 & 186,11 & 7,54 & 14,16 & 0,000 & 18,18 & 0,001 & 12,20 & 0,000 \\
\hline Cooper $217-0,845^{*}$ Edad & 186,93 & 10,67 & 187,81 & 12,22 & 186,50 & 9,63 & 14,65 & 0,000 & 18,85 & 0,001 & 12,59 & 0,000 \\
\hline Ellestad $197-0,556^{*}$ Edad & 177,22 & 7,02 & 177,79 & 8,37 & 176,93 & 6.34 & 4,93 & 0,083 & 8,84 & 0,111 & 3,02 & 0,378 \\
\hline Fernhall $205-0,64^{*}$ Edad & 182,23 & 8,08 & 182,89 & 9,64 & 181,90 & 7,30 & 9,94 & 0,000 & 13,94 & 0,012 & 7,99 & 0,010 \\
\hline Froelicher $207-0,64^{*}$ Edad & 184,23 & 8,08 & 184,89 & 9,64 & 183,90 & 7,30 & 11,94 & 0,000 & 15,94 & 0,004 & 9,99 & 0,001 \\
\hline Graettinger $199-0,63^{*}$ Edad & 176,58 & 7,95 & 177,24 & 9,49 & 176,26 & 7,18 & 4,30 & 0,132 & 8,28 & 0,135 & 2,35 & 0,505 \\
\hline Jones $210-0.65^{\circ}$ Edad & 186,87 & 8,20 & 187,55 & 9,79 & 186,84 & 7,41 & 14,59 & 0,000 & 18,59 & 0,001 & 12,93 & 0,000 \\
\hline Lester $205-0.41^{*}$ Edad & 190,41 & 5,18 & 190,84 & 6,17 & 190,20 & 4,67 & 18,13 & 0,000 & 21,88 & 0,000 & 16,29 & 0,000 \\
\hline 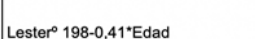 & 183,41 & 5,18 & 183,84 & 6,17 & 183,20 & 4,67 & 11,13 & 0,000 & 14,88 & 0,005 & 9,29 & 0,001 \\
\hline Morris $200-0,72^{*} \mathrm{Edad}$ & 174,38 & 9,09 & 175,13 & 10,84 & 174,02 & 8,21 & 2,10 & 0,538 & 6,17 & 0,285 & 0,11 & 0,890 \\
\hline Rodeheffer $214-1,00^{\circ} \mathrm{Edad}$ & 177,71 & 12,88 & 178,76 & 15,36 & 177,19 & 11,62 & 5,42 & 0,076 & 9,81 & 0,110 & 3,28 & 0,355 \\
\hline Sheffield $216-0,88^{*}$ Edad & 184,69 & 11,11 & 185,60 & 13,25 & 184,24 & 10,03 & 12,40 & 0,000 & 16,65 & 0,005 & 10,33 & 0,001 \\
\hline Whaley $209-0.7{ }^{*}$ Edad & 184,09 & 8,84 & 184,82 & 10,54 & 183,73 & 7,98 & 11,81 & 0,000 & 15,86 & 0,005 & 9,82 & 0,001 \\
\hline Whaley $214-0.8^{*}$ Edad & 185,53 & 10,10 & 186,36 & 12,05 & 185,12 & 9,12 & 13,25 & 0,000 & 17,41 & 0,002 & 11,21 & 0,000 \\
\hline Hossack 206-0.597"Edad & 184,76 & 7,54 & 185,38 & 8,99 & 184,45 & 6,81 & 12,47 & 0,000 & 16,42 & 0,003 & 10,54 & 0,000 \\
\hline sack & 189,03 & 13,47 & 190,14 & 16,07 & 188,49 & 12,16 & 16,75 & 0,000 & 21,19 & 0,001 & 14,58 & 0,000 \\
\hline
\end{tabular}

Tabla 4. Comparación de FCM por prueba de esfuerzo frente a ecuaciones predictivas en pacientes con obesidad y $4 \mathrm{FX}$ cardiovascular.

\begin{tabular}{|c|c|c|c|c|c|c|c|c|}
\hline & \multicolumn{2}{|c|}{ Menos 4 FX de Riesgo $(n=28)$} & \multicolumn{2}{|c|}{ Mayor 4 Fx'de Riesgo $(n=39)$} & \multicolumn{4}{|c|}{ FORMULA VS PRUEBA DE ESFUERZO } \\
\hline & Promedio & DE & Promedio & DE & Dif $<4 \mathrm{Fx}$ & Valor $p$ & Dif $>4 \mathrm{Fx}$ & Valor $p$ \\
\hline Prueba de Esfuerzo & 179,46 & 17,76 & 167,13 & 18,69 & & & & \\
\hline 220-Edad & 186,61 & 9,86 & 182,85 & 14,20 & 7,14 & 0,068 & 15,72 & 0,000 \\
\hline 210-Edad & 176,61 & 9,86 & 172,85 & 14,20 & $-2,86$ & 0,459 & 5,72 & 0,132 \\
\hline Tanaka (1997) 211-0,8* Edad & 184,29 & 7.89 & 181,28 & 11,36 & 4,82 & 0,232 & 14,15 & 0,000 \\
\hline Tanaka (2001) $207-0,7^{*}$ Edad & 183,63 & 6,90 & 180,99 & 9,94 & 4,16 & 0,305 & 13,86 & 0,000 \\
\hline Tanaka (2001) 206-0,7*Edad & 182,63 & 6,90 & 179,99 & 9,94 & 3,16 & 0,452 & 12,86 & 0,000 \\
\hline Tanaka (2001) 208,75-0,73*Edad & 184,37 & 7,20 & 181,63 & 10,36 & 4,91 & 0,222 & 14,50 & 0,000 \\
\hline Robinson (1938) 212-0,77*Edad & 186,29 & 7,59 & 183,39 & 10,93 & 6,82 & 0,088 & 16,26 & 0,000 \\
\hline Inbar 205,8-0,685"Edad & 182,93 & 6,76 & 180,35 & 9,72 & 3,46 & 0,413 & 13,22 & 0,000 \\
\hline Astrang $211-0,922^{*}$ Edad & 180,21 & 9,09 & 176,74 & 13,09 & 0,75 & 0,984 & 9,62 & 0,014 \\
\hline Bruce $210-0,662^{*}$ Edad & 187,89 & 6,53 & 185,40 & 9,40 & 8,43 & 0,032 & 18,28 & 0,000 \\
\hline Cooper $217-0,845^{\circ}$ Edad & 188,78 & 8,33 & 185,61 & 12,00 & 9,32 & 0,020 & 18,48 & 0,000 \\
\hline Ellestad 197-0,556"Edad & 178,43 & 5,48 & 176,34 & 7,89 & $-1,03$ & 0,641 & 9,21 & 0,009 \\
\hline Fernhall 205-0.64"Edad & 183,63 & 6,31 & 181,22 & 9,09 & 4,16 & 0,320 & 14,09 & 0,000 \\
\hline Froelicher 207-0,64*Edad & 185,63 & 6,31 & 183,22 & 9,09 & 6,16 & 0,123 & 16,09 & 0,000 \\
\hline Graettinger $199-0,63^{*}$ Edad & 177,96 & 6,21 & 175,59 & 8,94 & $-1,50$ & 0,589 & 8,46 & 0,018 \\
\hline Jones $210-0,65^{\circ}$ Edad & 188,29 & 6,41 & 185,85 & 9,23 & 8,83 & 0,022 & 18,72 & 0,000 \\
\hline Lester" 205-0.41*Edad & 191,31 & 4,04 & 189,77 & 5,82 & 11,84 & 0,001 & 22,64 & 0,000 \\
\hline Lester $198-0,41^{*}$ Edad & 184,31 & 4,04 & 182,77 & 5,82 & 4,84 & 0,214 & 15,64 & 0,000 \\
\hline Morris $200-0,72^{\circ}$ Edad & 175,96 & 7,10 & 173,25 & 10,22 & $-3,51$ & 0,281 & 6,12 & 0,106 \\
\hline Rodeheffer $214-1,02^{*} \mathrm{Edad}$ & 179,94 & 10,06 & 176,10 & 14,48 & 0,47 & 0,970 & 8,97 & 0,026 \\
\hline Sheffield 216-0,88* Edad & 186,61 & 8,68 & 183,30 & 12,49 & 7,15 & 0,082 & 16,18 & 0,000 \\
\hline Whaley $209-0,7^{*}$ Edad & 185,63 & 6,90 & 182,99 & 9,94 & 6,16 & 0,117 & 15,86 & 0,000 \\
\hline Whaley $214-0,8^{*}$ Edad & 187,29 & 7,89 & 184,28 & 11,36 & 7,82 & 0,047 & 17,15 & 0,000 \\
\hline Hossack 206-0,597"Edad & 186,06 & 5,89 & 183,82 & 8,48 & 6,60 & 0,089 & 16,69 & 0,000 \\
\hline Hossack 227-1,067"Edad & 191,37 & 10,52 & 187,36 & 15,15 & 11,91 & 0,005 & 20,23 & 0,000 \\
\hline
\end{tabular}




\section{DISCUSIÓN}

El propósito de este estudio fue comparar las ecuaciones de predicción para FCmáx frente a los resultados obtenidos en una prueba de esfuerzo mediante el protocolo de Bruce en pacientes con obesidad. Dicha investigación arrojó diferencias significativas y en muchas, sobreestimaciones realmente alarmantes, incluyendo las comparaciones según los factores de riesgo asociados a la obesidad. Diversos estudios, han manifestado diferencias de medias en las ecuaciones predichas en varios rangos de edad, sexo y antropometría. Zackary S., Oleg A. y Michael R. Esco et al. (2017) ${ }^{14}$ en deportistas demostraron que las formulas de Tanaka, Fox, Shargal y Nikolaidis; ninguna explica adecuadamente la variabilidad individual de la FCmáx y que los profesionales deben evitar aplicar estas ecuaciones.

Resultados similares a los encontrados en múltiples investigaciones sobre el tema, en Estados Unidos ${ }^{15}$ por la Universidad de Illinois en Chicago frente a las ecuaciones predictivas, en Brasil por la Universidad de Río Grande del Norte ${ }^{16}$ quienes demostraron una diferencia significativa en la fórmula 220-edad y que su uso es inadecuado para predecir el esfuerzo máximo. Al igual, que la Universidad Federal de Río de Jainero ${ }^{17}$. En Grecia ${ }^{18,19}$ por Hellenic Army Academy; Colombia ${ }^{20}$, con su trabajo colaborativo entre la Universidad Nacional y la Universidad del Bosque, quienes afirman una sobreestimación de dichas fórmulas y que no son predictores adecuados entre sujetos de 18 a 30 años de edad para la FCmáx durante el ejercicio en la altura de Bogotá. Además, de previas investigaciones realizadas por algunos de los autores de este trabajo, concluyen al igual que en este: "No se recomienda las ecuaciones evaluadas por su diferencia significativa respecto a la prueba de esfuerzo máxima; especialmente 220-Edad cuya diferencia de media estuvo por encima de 14 latidos por minutos" 21 .

Así mismo, todos los resultados anteriores y los nuestros, son corroborados por el National Institute Of Health en su publicación 2013 por Sarzynski M.A., Rankinen T., Earnest C.P., Leon A.S., Rao D.C. y Skinner J.S. et al. ${ }^{22}$ quienes concuerdan con nuestros resultados al mencionar que las ecuaciones no predicen con precisión la frecuencia cardiaca máxima medida para un individuo; como también, ineludiblemente los presentes resultados con llevan a recomendar que los médicos del deporte, fisioterapeutas, entrenadores físicos, licenciados en ciencias del deporte y entre otras profesiones encargadas de promocionar y prescribir ejercicio, deben tener en cuenta las diferencias encontradas en esta investigación; resaltando, que una inadecuada cifra obtenida de FCM generará errores al determinar los porcentajes de entrenamiento de nuestro paciente obeso e inadecuados para sus necesidades y por ende insatisfacción, frustración y desanimo por no alcanzar sus metas. Sugerencias similares a las redactas por Pantelis T. Nikolaidis, Thomas Rosemann y Beat Knechtle (2018). 


\section{CONCLUSIONES}

La presente investigación demuestra que las formulas predictivas para FCM en pacientes con obesidad son inadecuadas, debido a una sobreestimación de los valores reales; que en el peor de los casos pondrían generar un riesgo a la seguridad de los pacientes; además, otorgarían un valor erróneo para tomarlo como referencia para una adecuada prescripción del ejercicio.

\section{BIBLIOGRAFÍA}

1. Dávila-Torres J., González-Izquierdo J., Barrera-Cruz A. Panorama de la obesidad en México. Revista Médica Institucional Mexicana del Seguro Social. 2015;53(2):240249.

2. Barrera-Cruz A., Ávila-Jiménez L., Cano-Pérez E. et al. Prevención. Diagnóstico y tratamiento del sobrepeso y la obesidad exógena. Revista Médica Institucional Mexicana del Seguro Social.2013;51(3):344-357.

3. Córdova-Villalobos J., Barriguete-Meléndez J., Rivera-Montiel M. et al. (2010) Sobrepeso y obesidad. Situación actual y perspectivas. Acta Médica Grupo Ángeles.2019;8(4):202-207.

4. Rodrigo-Cano S., Soriano del Castillo J., Merino-Torres J. Causas y tratamiento de la obesidad. Nutrición clínica y dietética hospitalaria. 2107;37(4):87-92.

5. Barquera-Cervera S., Campos-Nonato I., Rojas R. et al. Obesidad en México: epidemiología y políticas de salud para su control y prevención. Gaceta Médica de México. 2010;146:397-407.

6. Cabrerizo L., Rubio M., Ballesteros M. et al. Complicaciones asociadas a la obesidad. Revista Española de Nutrición Comunitaria. 2008;14(3):156-162.

7. Arteaga A. El sobrepeso y la obesidad como un problema de salud. Revista Médica Clínica Condes. 2012;23(2):145-153.

8. López-Farré A., Macaya-Miguel C. Libro de la salud cardiovascular del hospital San Carlos y de la Fundación BBVA. 2009; Editorial Fundación BBVA. Bilbao.

9. Antúnez de la Rosa M., Jiménez y Villegas M., Cortés-Villarreal G. Valoración Inicial con prueba de esfuerzo al paciente cardiópata para ingresar al programa de rehabilitación cardíaca. Revista Mexicana de Enfermería Cardiológica. 2004;12(1):34-37.

10. Cruz-Cervantes R., González-Bravo F., Murillo-Ortíz B. et al. Empleo de la prueba de esfuerzo Bruce en una población de riesgo asintomática. Revista Mexicana de Cardiología. 2009;20(4):190-196.

11. Beistegui-Alejandre I., Sánchez-Carrio A. Manual de enfermería: Ergometría. 2013; Enfermería en Cardiología. Recuperado de: https://www.enfermeriaencardiologia. com/wp-content/uploads/cap_10_sec_05.pdf

12. Bouzas-Marins J., Ottoline-Marins N., Delgado-Fernández M. Aplicaciones de la frecuencia cardíaca máxima en la evaluación y prescripción de ejercicio. Apunts Medicina de Esport. 2010;45(168):251-258.

13. Buendia, R., Zambrano, M., Diaz, A., Reino, A., Ramirez, J., Espinosa, E. Puntos 
de corte de perímetro de cintura para el diagnóstico de obesidad abdominal en población colombiana usando bioimpedanciometría como estándar de referencia. Rev Colomb Cardiol. 2016;23(1):19-25.

14. Zackary S., Oleg A. y Esco R. Age-Predicted Maximal Heart Rate Equations Are Inaccurate for Use in Youth Male Soccer Players. 2017; Human Kinetics, Pediatric Exercise Science, (Ahead of Print) https://doi.org/10.1123/pes.2017-0281

15. Arena R., Myers J. y Kaminsky L. Revisiting age-predicted maximal heart rate: Can it be used as a valid measure of effort?. Am Heart J. 2016;173:49-56.

16. Oliveira H., Brandão N., Mendes G., Renee T., Ferreira V., Silva P. et al. Use of predictive equations of maximum heart rate for exercise prescription: a comparative study. Journal of Sports and Physical Education (IOSR-JSPE). 2016;3(1):04-08.

17. de Araújo C., Duarte C. Maximal heart rate in young adults: A fixed 188 bpm outperforms values predicted by a classical age-based equation, International Journal of Cardiology. 2015;1,(184):609-10. DOI: 10.1016/j.ijcard.2015.02.043.

18. Nikolaidis P. Maximal Heart Rate in Soccer Players: Measured versus Age $\square$ predicted. Biomed J. 2015;38:84-89.

19. Nikolaidis P., Padulo J., Chtourou H., Torres-Luque G., Heller J. Estimating Maximal Heart Rate With The '220-Age' Formula In Adolescent Female Volleyball Players: A Preliminary Study. Human Movement. 2014;15(3):166-170. DOI: 10.1515/humo2015-0007

20. Cruz-Martínez LE, Rojas-Valencia JT, Correa-Mesa JF, Correa-Mesa JC. Maximum Heart Rate during exercise: Reliability of the 220-age and Tanaka formulas in healthy young people in a moderate altitude. Rev Fac Med. 2014;62(4):579-85. http://dx.doi. org/10.15446/revfacmed.v62n4.44539.

21. Pereira Rodríguez, J., Boada Morales, L., Niño Rios, I., Cañizares Pérez, Y., Quintero Gómez. Frecuencia cardiaca máxima mediante 220 menos Edad versus prueba de esfuerzo con protocolo de Bruce. Movimiento Científico. 2017;11(1):15-22.

22. Sarzynski M.A., Rankinen T., Earnest C.P., Leon A.S., Rao D.C. y Skinner J.S. et al. Measured Maximal Heart Rates Compared to Commonly Used Age-Based Prediction Equations in the Heritage Family Study. Am J Hum Biol. 2013;25(5):695-701. DOI:10.1002/ajhb.22431. 